\begin{tabular}{|c|l|}
\hline Title & Very Singular Diffusion Equations Second and Fourth Order Problems \\
\hline Author(s) & Giga, Mi-Ho; Giga, Yoshikazu \\
\hline Citation & Hokkaido University Preprint Series in Mathematics, 960, 1-33 \\
\hline Issue Date & 2010-5-6 \\
\hline DOI & 10.14943/84107 \\
\hline Doc URL & http://hdl.handle.net/2115/69767 \\
\hline Type & bulletin (article) \\
\hline File Information & pre960.pdf \\
\hline
\end{tabular}

Instructions for use 


\title{
Very Singular Diffusion Equations - Second and Fourth Order Problems
}

\author{
Mi-Ho Giga and Yoshikazu Giga \\ Graduate School of Mathematical Sciences \\ University of Tokyo \\ Komaba 3-8-1, Meguro-ku \\ Tokyo, 153-8914, JAPAN
}

April 28,2010

\begin{abstract}
This paper studies singular diffusion equations whose diffusion effect is so strong that the speed of evolution becomes a nonlocal quantity. Typical examples include the total variation flow as well as crystalline flow which are formally of second order. This paper includes fourth order models which are less studied compared with second order models. A typical example of this model is an $H^{-1}$ gradient flow of total variation. It turns out that such a flow is quite different from the second order total variation flow. For example, we prove that the solution may instantaneously develop jump discontinuity for the fourth order total variation flow by giving an explicit example.
\end{abstract}

MSC 2010: 35K67, 35K92, 74N05, 62H35

Keywords: total variation flow, singular diffusion, fourth order model, jump discontinuity, image analysis, crystalline flow

\section{Introduction}

We are concerned with singular diffusion equations whose diffusion effect is so strong that the speed of evolution becomes a nonlocal quantity. Typical examples include the total variation flow as well as crystalline flow which 
are formally of second order. This paper includes fourth order models which is less studied compared with the second order model. A typical example of this model is an $H^{-1}$ gradient flow of total variation. It turns out that such a flow is quite different from usual total variation flow. This paper also includes a short survey for second order models although the references are not at all exhaustive.

Several examples are provided by image processing and materials science. We begin with two simple examples which are one dimensional version of total variation flow. One is the second order model formally written as

$$
u_{t}=\left(\operatorname{sgn} u_{x}\right)_{x}
$$

The other one is the fourth order model formally written as

$$
u_{t}=-\left(\operatorname{sgn} u_{x}\right)_{x x x} .
$$

Here we use the convention that subscripts denote partial derivatives, for example $f_{x x}=\partial^{2} f / \partial x^{2}, f_{t}=\partial f / \partial t$. Both are regarded as a 'gradient flow' of total variation energy $E(u)=\int\left|u_{x}\right| d x$ but the metric of differentiation is different. The first one is $L^{2}$-gradient flow while the second one is viewed as $H^{-1}$ gradient flow. The space $H^{-1}$ is considered as a dual space of the Sobolev space $H_{0}^{1}$. (We shall give a rigorous formulation in a later section for periodic boundary condition.) Although the equation is written as a form of partial differential equations, the speed is actually determined in nonlocal way, which causes a lot of problems even for second order models.

A good aspect of problems is that it is viewed as a gradient flow of a lower semicontinuous convex function. However, there are several difficult aspects since the energy density of total variation is not coercive (linear growth as $\left.\left|u_{x}\right| \rightarrow \infty\right)$ and it has a singularity at $u_{x}=0$. This last singularity leads the fact that the speed is determined in nonlocal way.

It is well-known under periodic boundary conditions the solution of (1.1) is Lipschitz in space if initial data is Lipschitz [FG]. In fact, there is a maximum principle for derivatives. However, for the fourth order problem, it turns out that the solution instantaneously may develop jump discontinuity even if the initial data is Lipschitz continuous. This can happen since our energy density is not coercive. We give an explicit example in this paper, which is a new contribution in this paper. We also show that the comparison principle is violated. 
For above two examples, the initial value problem is at least well-posed in the sense there exists a unique solution for a reasonably wide class of initial data since energy is a lower semicontinuous, convex function in a Hilbert space. (The solution is actually a global-in-time solution for these problems.) However, it often happens that well-posedness itself is not clear. We overview well-posedness problems for other examples which are not necessarily in the form of a gradient flow. There are already review articles [GG], [G0], [G1] on a similar topics so we mainly focus development after 2004 .

We consider a more general total variation flow equation for $u=u(t, x)$, $x=\left(x_{1}, \cdots, x_{n}\right)$ of the form

$$
u_{t}=\operatorname{div}(\nabla u /|\nabla u|)+\lambda(f-u),
$$

where $f$ is a given function and $\lambda \in \mathbf{R}$. Here $\nabla u$ represents the spatial gradient, i.e., $\nabla u=\left(\partial_{x_{1}} u, \ldots, \partial_{x_{n}} u\right), \partial_{x_{i}} u=\partial u / \partial x_{i}$. We also consider a more general version of (1.3) called an anisotropic mean curvature flow equation for the graph of a function $u$ of the form

$$
u_{t}=\sqrt{1+|\nabla u|^{2}} M(\vec{n})\left(\operatorname{div}\left(\left(\nabla_{p} W\right)(\nabla u)\right)+\sigma(x, u)\right),
$$

where $W=W(p)$ is a given convex function in $\mathbf{R}^{n}$ which may not be $C^{1}$ and $M(>0)$ is a given positive function; $\sigma$ is also a given function; $\vec{n}$ denotes the upward normal of the graph $y=u(t, x)$, i.e.,

$$
\vec{n}=\left(-\nabla u / \sqrt{\left(1+|\nabla u|^{2}\right)}, 1 / \sqrt{1+|\nabla u|^{2}}\right) .
$$

Here $\nabla_{p}$ denotes the gradient in $p$ variables.

Of course, (1.3) is a particular example of (1.4) by taking

$$
M(\vec{n})=1 / \sqrt{1+|\nabla u|^{2}}, W(p)=|p|
$$

and $\sigma(x, u)=\lambda(f(x)-u)$.

The total variation flow (1.3) can be formally viewed as an $L^{2}$-gradient flow of

$$
E(u)=\int_{\Omega}\left(|\nabla u|+\frac{\lambda}{2}(u-f)^{2}\right) d x,(\Omega: n-\text { dimensional manifold })
$$

i.e., $u_{t} \in-\partial E(u)$, where $\partial E$ denotes the $L^{2}$-subdifferential of $E$. If $\lambda \geq 0$, then $E$ is convex. Then there is a nice abstract theory for the existence 
of solutions for the initial value problem $\partial_{t} u \in-\partial E(u)$ when $E$ is a lower semicontinuous convex function defined in a Hilbert space initiated by $\mathrm{Y}$. Kōmura [Ko] and developed by H. Brezis and others in 1960s see e.g. [B]. For our problem (1.3) it guarantees the unique solvability of the initial value problem for (1.3) with $\lambda \geq 0$ under for example periodic boundary condition (by taking $\Omega=\mathbf{T}^{n}$, a flat torus) with $f \in L^{2}(\Omega)$. Surprisingly, the speed (the right time derivative) is determined uniquely although the evolution law $u_{t} \in-\partial E(u)$ looks ambiguous. In fact, the speed is determined by a minimal section or canonical restriction of $\partial E(u)$. However, its explicit characterization is often nontrivial [AD] and it is well-studied for piecewise constant functions $[\mathrm{ACM}],[\mathrm{ABCM}],[\mathrm{Mol}],[\mathrm{GGK}],[\mathrm{SK}],[\mathrm{S}] \ldots$ For development of the theory for total variation flow the reader is referred to a nice book [ACM] and references therein. For an intuitive explanation of the theory see [KG].

The corresponding fourth order problem formally written as

$$
u_{t}=-\Delta[\operatorname{div}(\nabla u /|\nabla u|)+\lambda(f-u)]
$$

is also formulated in the framework of subdifferential equation $u_{t} \in-\partial_{H^{-1}} E(u)$, where subdifferential is taken in $H^{-1}$ norm. Of course (1.2) is a particular example of (1.6). The general theory guarantees its unique solvability. However, to determine the speed we need to calculate a subdifferential which is not easy in general. See Y. Kashima [Ka], where slightly different problem is studied. The reader is referred to [GKohn] for a formulation when $\lambda=0$.

To see that the speed is a nonlocal quantity we consider (1.1) which is one-dimensional version of (1.3) with $\lambda=0$. If one considers $u_{0}(x)=\cos x$ as an initial data, it is expected that a flat part instantaneously develops from maximum point and minimum point. The other part is not expected to move. Heuristically, the place where slope equals zero feels very strong diffusion while other part stops. In fact, the unique solution provided by the abstract theory is of the form

$$
u(x, t)= \begin{cases}\cos x & d(t) \leq|x| \leq \pi / 2, \\ \cos d(t) & |x| \leq d(t)\end{cases}
$$

and $d(t)$ is determined by

$$
u_{t}(x, t)=2 /(\text { length of flat part })(=2 / 2 d(t)),|x|<d(t)
$$

with $d(0)=0$. This fact is observed for example by T. Fukui and Y. Giga [FG] and R. Hardt and X. Zhou [HZ] a long time ago. It is clear that the speed is determined by a nonlocal quantity. 
If we consider (1.4), it cannot be viewed as a gradient flow so general theory for $u_{t} \in-\partial E(u)$ does not apply. The notion of a solution itself should be studied. A viscosity like theory [CIL] developed by M.-H. Giga and Y. Giga [GG98Ar], [GG99], [GG01Ar] during 1998-2001 covers the case that the space dimension $n$ is one and $\sigma$ equals a constant with some technical assumptions on $W$ (which applies a convex, piecewise linear function $W$ ). In particular, the initial value problem for (1.4) is well-posed if $\sigma$ is constant. The underlying structure of their theory is that flat position (with slope corresponding to a jump of $W$ ) stays as flat so that the speed is constant. This hypotheses (which is verified by subdifferential theory if it applies) is also fundamental to construct admissible crystalline flows by J. Taylor [T1], and independently by S. B. Angenent and M. E. Gurtin [AG]. Actually, the speed is determined by solving an obstacle problem [KS] which may not be a constant when the driving force $\sigma$ in (1.4) is not spatially constant (as observed in the work of [GG98DS]) or the space dimension $n \geq 2$ (as observed by G. Bellettini M. Novaga and M. Paolini [BNP]).

In fact, for surface evolution [G2] by singular energy $\gamma$ of the form

$$
V=\gamma \operatorname{div}_{\Gamma}\left(\nabla_{p} \gamma\right)(\vec{n}),
$$

where $\gamma$ is an interfacial energy and $V$ is the normal velocity of an evolving hypersurface. Here $\operatorname{div}_{\Gamma}$ denotes the surface divergence. G. Bellettini and M. Novaga introduced a notion of solution and established uniqueness result [BN]. However, the existence of solution is known only for convex initial data [BCCN]. Flat portion such that the speed is constant is called a caribrable set for $E$ or a Cheeger set studied by people including B. Kawohl [KSch], [KL]. Its relation of the Rayleigh quotient of 1-Laplacian i.e., $\int|\nabla u| d x / \int|u| d x$ is well-studied. It is interesting to charaterize caribrable sets in a general setting [BNP1](cf. [BNP2]).See [BM] for a review of anisotropic flow, where 4th order problem like anisotropic Willmore flow is derived.

The case when $\sigma$ in (1.4) is not constant is handled recently by M.H. Giga Y. Giga and P. Rybka [GGiR], where they study dependence of solutions of the obstacle problems with respect to the domain (flat portion) we consider to obtain a comparison principle. However, the analysis is still limited for one-dimensional problem. Nevertheless, this is a fundamental key to develop a level set theory [CGG], [ES], [G3] for all planer singular curvature flow including crystalline flow with nonuniform driving force term. There is another approach to construct an explicit solution by solving a free 
boundary problem ([GGR], [GR4], [GR5]). We also note that the speed agrees with the speed proposed by $\mathrm{A}$. R. Roosen $[\mathrm{R}]$.

Well-posedness problem is widely open for 1-harmonic map flow:

$$
u_{t}=\operatorname{div}\left(\frac{\nabla u}{|\nabla u|}\right)+|\nabla u| u,
$$

where $u$ is a mapping to a unit sphere in $\mathbf{R}^{m}$. It is solvable locally-in-time [GKY]. However, there is a chance that the solution may break down in finite time like usual harmonic map flow. This is proved by [GKu] for a rotationally symmetric 1-harmonic map flow from disk to the sphere $S^{2}$. The theory is further developed by [Gia M]. A global existence of weak solutions is recently established by J. W. Barrett, X. Feng and A. Prohl [BFP]. However, its uniqueness is not known even locally (except one dimensional case see Y. Giga and R. Kobayashi [GK]).

In the next section we briefly survey several sources of problems. In Section 3 we give an explicit solution of 4th order total variation flow and prove that it may lose continuity instantaneously even if the initial data is Lipschitz or even smooth. Also, we show that the comparison principle may be violated.

\section{Source of Problems}

\subsection{Image Analysis}

The energy (1.5) involving total variation is first introduced by [ROF] to recover an image, where $f$ is a given gray-scale image with fidelity constant $\lambda>0$. The minimizer $u$ of this energy is regarded as 'a recovered image' from $f$. This problem is by now well-analyzed for example in [CL]. The book [ACM] includes a nice introduction of this topic. Clearly, the gradient flow (1.3) is a good way to find the minimizer of (1.5).

The fourth order model is proposed by [OSV]. Instead of minimizing $E$ in (1.5), they proposed to minimize

$$
F(u)=\int_{\Omega}|\nabla u|+\frac{\lambda}{2}\|u-f\|_{H^{-1}}^{2} .
$$

Its $H^{-1}$ gradient flow (with the Neumann boundary condition) is (1.6). This is studied by [ElS], where they proved well-posedness by the Galerkin method as well as for regularized problems; see also [EIS2] for its numerical analysis. 
The 1-harmonic map flow is introduced in image analysis for denoising color image [TSC], [VO]. Let $I$ be a vector valued function

$$
I(x)=(r(x), g(x), b(x))
$$

at each pixel $x=\left(x_{1}, x_{2}\right) \in \Omega \subset \mathbf{R}^{2}$. This function represents the intensity of the three primary colors (red, green and blue). The chromaticity is defined by

$$
u=I(x) /|I(x)|
$$

while the brightness is defined by $|I(x)|$. The main idea is to decompose the image into chromaticity and the brightness and denoise both separately using a different method. To recover chromaticity one way is to use total variation like energy $E$ in (1.5). However, now our $u$ is $S^{2}$-valued. So the corresponding gradient flow is now 1-harmonic map flow. If $\lambda=0$, its explicit form is $(1.8)$.

\subsection{Materials Science}

The equation (1.4) is the evolution equation of the graph of a function moved by the crystalline energy when $W$ is a convex, piecewise linear function. A crystalline flow of the form (1.7) was first proposed by [AG] and [T1] for planar motion to describe a motion of phase boundaries. (In this case the interfacial energy $\gamma$ is assumed to be a positively homogeneous function of degree one with convex polygonal sublevel set $\gamma \leq 1$ (called a Frank diagram).) One merit is that if one restricts a motion for a special class called admissible, the evolution is reduced to a system of ordinary differential equations. By a crystalline flow we often mean such a special flow. For the current status of the crystalline flow the reader is refereed to [I] and references therein; see also [GGH] and [GGO]. Its higher dimensional version was first studied by [GGM]. They constructed a crystalline flow for polyhedra; however, they overstated that this flow enjoys comparison principle. Later, it turns out that there is a facet whose speed is not constant [BNP] if one admits the comparison principle. See also $[\mathrm{GPR}]$ and $[\mathrm{BCCN}]$ for a formulation of the problem for general interfacial energy.

In materials science interfacial energy has a singularity in low temperature so the equilibrium shape, the Wulff shape has a flat part called a facet. Such an energy is very important to handle the problem as a continuous macroscopic thermodynamics. 
From this point of view it is quite natural to consider the fourth order model, a relaxation dynamics. For example, fourth order crystalline motion is formulated and calculated by [CRCT] as a special model of surface diffusion phenomena. The equation $V=-\Delta_{\Gamma} H$ with mean curvature $H$ is called a surface diffusion law, where $\Delta_{\Gamma}$ denotes the surface Laplacian. It goes back to Mullins $[\mathrm{M}]$ and several mathematical results will be discussed in Section 4. See $[\mathrm{CT}]$ for a review.

Other than crystalline surface diffusion flows it is also proposed to use a model of describe a growing surface in low temperature [Sp]. As discussed in Remark(iii) of Section 3.1 the $H^{-1}$ gradient flow of $\Phi$ is of the form

$$
u_{t}=-\Delta\left[\operatorname{div}(\nabla u /|\nabla u|)+\mu \operatorname{div}\left(|\nabla u|^{q-2} \nabla u\right)\right] .
$$

Here $u$ represents the height of a crystal surface. It is analysed by [Ka] and $[\mathrm{O}]$. According to the observation by [O], this evolution seems not the same as a continuum limit of step motion dynamics.

Another important source is provided to model an evolution of multigrains proposed by [KWC]: for preliminary study of the problem see [KG] and [GGK]. It is a total variation flow for mean orientation of each grain coupled with other order parameter. Its explicit form is

$$
\begin{aligned}
\tau_{\eta} \eta_{t} & =\kappa \Delta \eta+1-\eta-2 s \eta|\nabla \theta|, \\
\eta^{2} \tau_{0} \theta_{t} & =s \operatorname{div}\left(\eta^{2} \nabla \theta /|\nabla \theta|\right)+\nu \Delta \theta
\end{aligned}
$$

Here the unknown variable $\theta$ is an indicator of the mean orientation of the crystalline and another unknown variable $\eta$ is an order parameter for the degree of crystalline orientation. The quantities $\tau_{\eta}, \tau_{0}, \kappa, s$ are positive constant. The constant $\nu$ is nonnegative and can be zero. Recently, the initial value problem with $\nu>0$ under the Neumann condition is solved globally-in-time in a smoothly bounded domain in $\mathbf{R}^{n}$. In fact, a global-in-time week solution is constructed in [IKY2]. If $\eta^{2}$ in front of $\theta_{t}$ is replaced by a strictly positive function having a similar property, a global-in-time weak solution is constructed in [IKY1] and the uniqueness is given for one-dimension. Existence and uniqueness of one dimensional model with $\eta_{x x}$ replaced by $\left(\sigma \eta_{t}+\kappa \eta\right)_{x x}$ has been established in [IGNS]. However, it is an open problem whether or not the problem is solved for $\nu=0$. Also it is more natural that $\theta$ is a function with values in $S^{n-1}$. In particular, if we consider the planer case it is natural that $\theta$ is $S^{1}$ valued. Since the mean orientation function should 
be considered as $S^{1}$ valued, it gives another source of 1-harmonic map flow [GK].

\subsection{Vertical Diffusion}

Although there are several applications we just give here rather unusual applications. It is summarized as a application of vertical singular diffusion to have solutions with shocks.

We consider the graph $\Gamma_{t}$ of solution $u$ of the Burgers equation

$$
u_{t}+u u_{x}=0 \text {. }
$$

If we use upward normal velocity $V$ of $\Gamma_{t}$ in $\mathbf{R}^{2}=\{(x, y) \mid x, y \in \mathbf{R}\}$ it is formally of the form:

$$
V=y \text { on } \Gamma_{t}
$$

However, the solution of (2.2) may overturn and may not be the graph of a function. For (2.1) we often consider an entropy solution with jump discontinuities instead of a multivalued (overturned) solution. What is a good equation instead of (2.2) to track an entropy solution? Singular diffusion equation gives an answer as rigorously discussed by M.-H. Giga-Y. Giga [GG03]. The main idea is to consider

$$
V=y+A \operatorname{div}(\nabla \gamma(\vec{n})),
$$

where $\gamma\left(p_{1}, p_{2}\right)=\left|p_{2}\right|$ and $A>0$ instead of (2.2). It is useful to calculate numerically (cf. T.-H. R. Tsai, Y. Giga and S. Osher [TGO]). The last term of (2.3) is called a vertical singular diffusion term. If $A>0$ is taken sufficiently large with respect to size of jumps (but still finite), then the solution of (2.3) is the graph of entropy solutions. This method works to calculate entropy solutions for multidimensional scalar conservation law [TGO]. However, its application is not limited to conservation laws. It applies to calculation of a unique proper viscosity solution [G2] with shocks.

The unique existence of global entropy solutions is established a long time ago by S. N. Kruzhkov $[\mathrm{Kr}]$ for $L^{\infty}$ initial data. His idea was extended to several other problems. For example, J. Carrillo [C] showed the existence and uniqueness of entropy solutions to nonlinear strongly degenerate parabolic equations under the homogeneous Dirichlet boundary condition. More recently, H. Watanabe and S. Oharu [WO] formulated BV-entropy solutions 
for the Neumann problem and proved the unique existence of global BVentropy solutions. This type of idea is also important to construct a unique global solution to the total variation flow (1.3) when initial data is in $L^{1}$ [ACM].

For scalar conservation laws in multidimensional spaces there are several ways to approximate entropy solutions by solving linear equations in the graph spaces and taking average in the vertical variable. This idea is called a kinetic construction or transport-collapse approximation. It goes back to works of [GM], [GMO], [B1], [B2], [Mi]. Very recently, Y. Brenier [B3] recovered the Kruzhkov entropy solution from the solution of a subdifferential equation in the graph space with indicator function preventing overturning without any approximation.

It is curious whether such singular diffusion can be used for sand pile problems studied by L. C. Evans, M. Feldman and R. F. Gariepy [EFG]. If the slope bound is 1 i.e., $|\nabla u| \leq 1$, a suggestive equation is

$$
u_{t}=f(x)+A \int_{|\omega|=1}(\operatorname{sgn}(1-\nabla u \cdot \omega))_{x} d \omega, A>0,
$$

when the original equation is $u_{t}=f(x)$.

\subsection{Approximation}

It is important to develop several ways of approximation of solutions not only for construction of a solution but also to study properties of a solution. In fact, Bellettini and et al [BCCN] constructed crystalline surface evolution flow by using Almgren-Taylor-Wang [ATW] scheme for convex initial data.

General tendency is that various approximation is uniform with respect to mollification way of singular interfacial energy. This is actually proved for the anisotropic Allen-Cahn equation by Y. Giga, T. Ohtsuka and R. Schätzle [GOS] when the driving force term is spatially homogeneous but for arbitrary dimension. It is interesting to study recent differential game approximation by R. V. Kohn and S. Serfaty [KS1], [KS2] of a solution although approximation scheme for singular interfacial energy is not yet given. For example it is interesting to give a differential game interpretation for crystalline curvature flow.

For fourth order problem less is known. An extension of Almgren-TaylorWang scheme is proposed but it is an open problem to characterize its limit [CT], [Chu]. 


\section{Total Variation Flow in a Sobolev Space of Negative Order}

In this section we give an explicit example that a solution for (1.2) may develop jump discontinuity instantaneously. We also give an example that the comparison principle is violated. These examples clarify the difference between (1.1) and (1.2).

\subsection{Loss of Continuity}

We consider (1.2) with periodic boundary condition. We have to find a rigorous formulation of the problem. Let $H^{1}(\mathbf{T})$ denote the Sobolev space of real-valued periodic function with period $\omega$ equipped with inner product

$$
(f, g)_{H^{1}}=((f, g))_{1}+\int_{\mathbf{T}} f \cdot g d x, f, g \in H^{1}(\mathbf{T}), \mathbf{T}=\mathbf{R} / \omega \mathbf{Z}
$$

here

$$
((f, g))_{1}=\int_{\mathbf{T}} f_{x} \cdot g_{x} d x .
$$

We consider a subspace so that $((f, g))_{1}$ becomes an equivalent inner product. Let $H_{\mathrm{av}}^{1}(\mathbf{T})$ be the codimension one subspace of the form

$$
H_{\mathrm{av}}^{1}(\mathbf{T})=\left\{f \in H^{1}(\mathbf{T}) \mid \int_{\mathbf{T}} f d x=0\right\},
$$

i.e., the space of $H^{1}$ functions whose average equals zero. By the Poincaré inequality or Wirtinger inequality it is easy to see that $((f, g))_{1}$ is an equivalent inner product in $H_{\mathrm{av}}^{1}(\mathbf{T})$ which is a closed subspace of $H^{1}(\mathbf{T})$. The dual space $H_{\mathrm{av}}^{-1}(\mathbf{T})$ is regarded as a subspace of the space $\mathcal{D}^{\prime}(\mathbf{T})$ of Schwartz' distributions. Let $\langle\cdot, \cdot\rangle$ denotes the canonical pairing of $H^{1}$ and $H^{-1}$, i.e. for $g \in H^{-1}(\mathbf{T})$ its value $g(f)$ at $f \in H^{1}(\mathbf{T})$ is denoted by

$$
g(f)=\langle f, g\rangle .
$$

It is formally written as $\int_{\mathbf{T}} f \cdot g d x$. It is identified with

$$
H_{\mathrm{av}}^{-1}(\mathbf{T})=\left\{f \in H^{-1}(\mathbf{T}) \mid\langle 1, f\rangle=\int_{\mathbf{T}} f d x=0\right\},
$$


where $H^{-1}(\mathbf{T})$ is the dual space of $H^{1}(\mathbf{T}) \subset \mathcal{D}^{\prime}(\mathbf{T})$. This space $H_{\mathrm{av}}^{-1}(\mathbf{T})$ is the Hilbert space equipped with an inner product

$$
((f, g))_{-1}=\left\langle(-\Delta)^{-1} f, g\right\rangle=\int_{\mathbf{T}}(-\Delta)^{-1} f \cdot g d x
$$

where $-\Delta$ denotes an isometry from $H_{\mathrm{av}}^{1}(\mathbf{T})$ to $H_{\mathrm{av}}^{-1}(\mathbf{T})$ defined by $f \mapsto$ $((f, \cdot))_{1}$. It is regarded as the Laplacian.

We are now ready to formulate (1.2) with periodic boundary condition in a rigorous way. We consider a functional

$$
\Phi_{\pi}(v)= \begin{cases}\int_{\mathbf{T}}\left|v_{x}\right|, & v \in B V(\mathbf{T}) \cap H_{\mathrm{av}}^{-1}(\mathbf{T}), \\ \infty, & \text { otherwise }\end{cases}
$$

in $H_{\mathrm{av}}^{-1}(\mathbf{T})$. Note that we always assume that $\int_{\mathbf{T}} v d x=0$ when the energy $\Phi_{\pi}(v)$ is finite. The functional $\int_{\mathbf{T}}\left|v_{x}\right|$ is the total variation defined by

$$
\int_{\mathbf{T}}\left|v_{x}\right|=\sup \left\{\int_{\mathbf{T}} \varphi_{x} v d x \mid\|\varphi\|_{L^{\infty}(\mathbf{T})} \leq 1, \varphi \in C^{1}(\mathbf{T})\right\}
$$

The space $B V(\mathbf{T})$ is the space of $L^{1}(\mathbf{T})$-function whose total variation is finite.

We consider a gradient flow equation of the form

$$
\begin{aligned}
& \frac{d u}{d t}(t) \in-\partial_{H^{-1}} \Phi_{\pi}(u(t)) \quad \text { a.e. } t>0 \\
& \left.u\right|_{t=0}=u_{0} \in H_{\mathrm{av}}^{-1}(\mathbf{T})
\end{aligned}
$$

where $\partial_{H^{-1}}$ denotes the subdifferential of $\Phi_{\pi}$ in $H_{\mathrm{av}}^{-1}(\mathbf{T})$. It is defined as

$$
\begin{aligned}
& \partial_{H^{-1}} \Phi_{\pi}(v)= \\
& \left\{f \in H_{\mathrm{av}}^{-1}(\mathbf{T}) \mid \Phi_{\pi}(v+h)-\Phi_{\pi}(v) \geq((h, f))_{-1} \text { for all } h \in H_{\mathrm{av}}^{-1}(\mathbf{T})\right\} .
\end{aligned}
$$

Since $\Phi_{v}$ is lower semicontinous and convex, an abstract theory (initiated by Komura [Ko] see [B]) yields a unique solution $u \in C\left([0, \infty), H_{\mathrm{av}}^{-1}(\mathbf{T})\right)$ of $(3.1)$ which is absolutely continuous in any compact interval in $(0, \infty)$ with values in $H_{\mathrm{av}}^{-1}(\mathbf{T})$. Moreover, $t \mapsto u(t)$ is right differentiable for all $t>0$ and

$$
\frac{d^{+} u}{d t}(t)=-\partial_{H^{-1}}^{0} \Phi_{\pi}(u(t))
$$


for all $t>0$, where $\partial_{H^{-1}}^{0} \Phi_{\pi}$ is the minimal section (or canonical restriction) of subdifferential $\partial_{H^{-1}} \Phi_{\pi}(v)$, i.e.,

$$
\partial_{H^{-1}}^{0} \Phi_{\pi}(v)=\operatorname{argmin}\left\{\|f\|_{H^{-1}} \mid f \in \partial_{H^{-1}} \Phi_{\pi}(v)\right\} .
$$

We shall explain why (3.1) represents (1.2) at least in a formal level. By recalling definition of subdifferential (3.2) we easily guess that $f \in \partial_{H^{-1}} \Phi_{\pi}(v)$ is equivalent to saying that $(-\Delta)^{-1} f \in \partial \Phi_{\pi}(v)$, where $\partial \Phi_{\pi}(v)$ is $L^{2}$-subdifferential of $\Phi_{\pi}$ since $((h, f))_{-1}=\int_{\mathbf{T}} h \cdot(-\Delta)^{-1} f d x$. Thus $\frac{d u}{d t} \in-\partial_{H^{-1}} \Phi_{\pi}(u)$ is formally equivalent to

$$
(-\Delta)^{-1} \frac{d u}{d t} \in-\partial \Phi_{\pi}(v)
$$

Since (an element of) $-\partial \Phi_{\pi}$ is formally equals $\operatorname{div}(\nabla u /|\nabla u|)=\left(\operatorname{sgn} u_{x}\right)_{x}$, operating $-\Delta$ both sides to get (1.2) at least formally.

In general it is difficult to characterize a subdifferential of a functional. However, for particular geometry of $v$ it is possible to characterize at least $L^{2}$ subdifferentials $\partial \Phi_{\pi}(v)$. For general characterization see [ACM]. We first recall a relation of $\partial_{H^{-1}}$ and $\partial$ which is formally discussed in the previous paragraph. Let $L_{\mathrm{av}}^{2}(\mathbf{T})$ denote the space of all $L^{2}(\mathbf{T})$ function whose average equals zero.

Lemma 3.1. ([GKohn]) Assume that $v \in L_{\mathrm{av}}^{2}(\mathbf{T})$. Then $f \in \partial_{H^{-1}} \Phi_{\pi}(v)$ if and only if $(-\Delta)^{-1} f \in \partial \Phi_{\pi}(v)$. Here $\partial \Phi_{\pi}(v)$ is the $L^{2}$-subdifferential of $\Phi_{\pi}$ at $v$, i.e.,

$$
\partial \Phi_{\pi}(v)=\left\{g \in L_{\mathrm{av}}^{2}(\mathbf{T}) \mid \Phi_{\pi}(v+h)-\Phi_{\pi}(v) \geq \int_{\mathbf{T}} h g d x \text { for all } h \in L_{\mathrm{av}}^{2}(\mathbf{T})\right\} .
$$

We consider a special profile of initial data $u_{0}(x)$ such that

$$
u_{0}(x)= \begin{cases}h_{0}, & |x|<r_{0} \text { with } 0<r_{0}<\omega / 4 \\ v_{0}(x), & r_{0} \leq|x|<\omega / 4\end{cases}
$$

where $v_{0} \in C^{1}\left[-\omega_{0} / 4, \omega_{0} / 4\right]$ is an even function strictly decreasing in $x \in$ $[0, \omega / 4]$ and $v_{0}(\omega / 4)=0$. The constant $h_{0}>0$ is assumed to satisfy $h_{0} \geq v_{0}\left(r_{0}\right)$. We extend this function on $(\omega / 4, \omega / 2)$ so that $u_{0}\left(\frac{\omega}{4}+x\right)=$ $-u_{0}\left(\frac{\omega}{4}-x\right), 0<x<\frac{\omega}{2}$ (as an odd function with respect to $x=\frac{\omega}{4}$.) We further extend this function so that $u_{0}$ is periodic with period $\omega$. Of course, 
it is easy to observe that $\int_{0}^{\omega} u_{0} d x=0$, i.e., $u_{0} \in L_{\mathrm{av}}^{2}(\mathbf{T})$. For this particular profile of $u_{0}$ one is able to calculate $\partial \Phi_{\pi}\left(u_{0}\right)$.

Lemma 3.2. Assume that $h_{0} \geq v_{0}\left(r_{0}\right)$. For $u_{0}$ in (3.4) a function $f \in L_{\mathrm{av}}^{2}(\mathbf{T})$ belongs to subdifferential $\partial \Phi_{\pi}\left(u_{0}\right)$ if and only if for $x \in(-\omega / 4,3 \omega / 4)$

$$
f(x)= \begin{cases}-\eta_{x}, & |x| \leq r_{0} \\ -\zeta_{x}, & \omega / 2+r_{0} \geq|x| \geq \omega / 2-r_{0}, \\ 0, & \text { otherwise }\end{cases}
$$

with some $\eta \in H^{1}\left(-r_{0}, r_{0}\right)$ satisfying

$$
\eta\left(-r_{0}\right)=1, \eta\left(r_{0}\right)=-1 \text { and }|\eta| \leq 1 \text { on }\left(-r_{0}, r_{0}\right),
$$

and some $\zeta \in H^{1}\left(\omega / 2-r_{0}, \omega / 2+r_{0}\right)$ satisfying

$$
\begin{aligned}
& \zeta\left(\omega / 2-r_{0}\right)=-1, \zeta\left(\omega / 2+r_{0}\right)=1 \\
& \text { and }|\zeta| \leq 1 \text { on }\left(\omega / 2-r_{0}, \omega / 2+r_{0}\right) .
\end{aligned}
$$

This lemma can be proved by using the following characterization (Lemma 3.3) together with Lemma 3.1. Lemma 3.3 is a trivial modification of [[ACM], Proposition 1.10], which is stated for the Neumann Problem.

Lemma 3.3. For general $w \in L_{\mathrm{av}}^{2}(\mathbf{T})$ a function $f \in L_{\mathrm{av}}^{2}(\mathbf{T})$ belongs to $\partial \Phi_{\pi}(w)$ if and only if there is a function $\xi \in L^{\infty}(\mathbf{T}) \cap H^{1}(\mathbf{T})$ with $f=-\xi_{x}$ such that $|\xi| \leq 1$ on $\mathbf{T}$ and that $\int_{\mathbf{T}} f \cdot w d x=\int_{\mathbf{T}}\left|w_{x}\right| d x$.

One merit of this characterization is that it is easy to extend to multidimensional situation. Also it is rather easy to see that for $u_{0}$ in Lemma 3.2 with $h_{0}<v_{0}\left(r_{0}\right)$ the subdifferential $\partial \Phi_{\pi}\left(u_{0}\right)$ is empty. Of course, one can directly prove Lemma 3.2 in the line of ([GGK], Lemma 1$)$, where $u_{0}$ is a piecewise constant function.

We are now ready to calculate the profile of solution of (3.1) (or (3.3)) with initial data $u_{0}$. As a result we are able to claim that the solution may instantaneously develop discontinuities. This phenomenon does not occur for $L^{2}$-total variation flow.

Theorem 3.4. Assume that $h_{0}=v_{0}\left(r_{0}\right)$ so that $u_{0}$ is at least Lipschitz continuous. Assume that $v_{0} \in C^{1}[0, \omega / 4]$ and $v_{0}^{\prime}<0$ on $(0, \omega / 4]$ with $v_{0}(\omega / 4)=0$. Then the solution $u$ of (3.1) with initial data $u_{0}$ instantaneously becomes discontinuous for $t>0$ (until it identically equals zero in finite time). 
The rest of this section is devoted to the proof of this main result (Theorem 3.4). We first calculate the minimal section of $\partial_{H^{-1}} \Phi_{\pi}(v)$ to derive an explicit formula of the solution. Let $\delta(x)$ denote the Dirac delta function.

Lemma 3.5 (Minimal section). Assume that $h_{0} \geq v_{0}\left(r_{0}\right)$. If $u_{0}$ has a profile given in (3.4), then

$$
\begin{aligned}
\partial_{H^{-1}}^{0} \Phi_{\pi}\left(u_{0}\right)(x) & \\
= & \frac{24}{L^{3}} \chi_{\left(-r_{0}, r_{0}\right)}+\frac{12}{L^{2}}\left(\delta\left(x-\omega / 2+r_{0}\right)+\delta\left(x-\omega / 2-r_{0}\right)\right) \\
& -\frac{12}{L^{2}}\left(\delta\left(x+r_{0}\right)+\delta\left(x-r_{0}\right)\right)-\frac{24}{L^{3}} \chi_{\left(\omega / 2-r_{0}, \omega / 2+r_{0}\right)}
\end{aligned}
$$

for $x \in(-\omega / 4,3 \omega / 4)$. Here $L$ is the length of flat part (called a facet) so that $L=2 r_{0}$ and $\chi_{(\alpha, \beta)}$ is the characteristic function of the interval $(\alpha, \beta)$.

Proof. We set $F=\partial_{H^{-1}}^{0} \Phi_{\pi}\left(u_{0}\right)$. By Lemma 3.1 and Lemma 3.2 we observe that $F=-\bar{f}_{x x}$ with

$$
\bar{f}=\operatorname{argmin}\left\{\left\|f_{x x}\right\|_{H_{\mathrm{av}}^{-1}(\mathbf{T})} \mid f \text { is of the form }(3.5)\right\} .
$$

Since

$$
\left\|f_{x x}\right\|_{H_{\mathrm{av}(\mathbf{T})}^{-1}}=\left\|f_{x}\right\|_{L^{2}(\mathbf{T})}
$$

it suffices to minimize

$$
\left\|\eta_{x x}\right\|_{L^{2}\left(-r_{0}, r_{0}\right)} \text { and }\left\|\zeta_{x x}\right\|_{L^{2}\left(\omega / 2-r_{0}, \omega / 2-r_{0}\right)}
$$

with (3.6) and (3.7) and the continuity of $f$ at $\pm r_{0}, \omega / 2 \pm r_{0}$, which are end points of facets. The last condition is required since $f_{x}$ is in $L^{2}(\mathbf{T})$. Since $\zeta$ can be treated in a parallel way, we just study $\eta$. The minimizing problem we have to solve is an obstacle problem. We have to find $\eta \in H^{2}\left(-r_{0}, r_{0}\right)$ which minimizes

$$
\int_{-r_{0}}^{r_{0}}\left|\eta_{x x}\right|^{2} d x
$$

with the constraint $|\eta(x)| \leq 1$ in $\left(-r_{0}, r_{0}\right)$ and boundary conditions $\eta\left(-r_{0}\right)=$ $1, \eta\left(r_{0}\right)=-1$ and $\eta_{x}\left( \pm r_{0}\right)=0$. This is still a lower semicontinuous (in $H^{2}$ ), strict convex minimizing problem so there is a unique minimizer. In the place where $|\eta(x)|<1$, the minimizer should satisfies the Euler-Lagrange equation $\left(\eta_{x x}\right)_{x x} \equiv 0$ so it must be a polynomial of degree 3 . If one finds a 


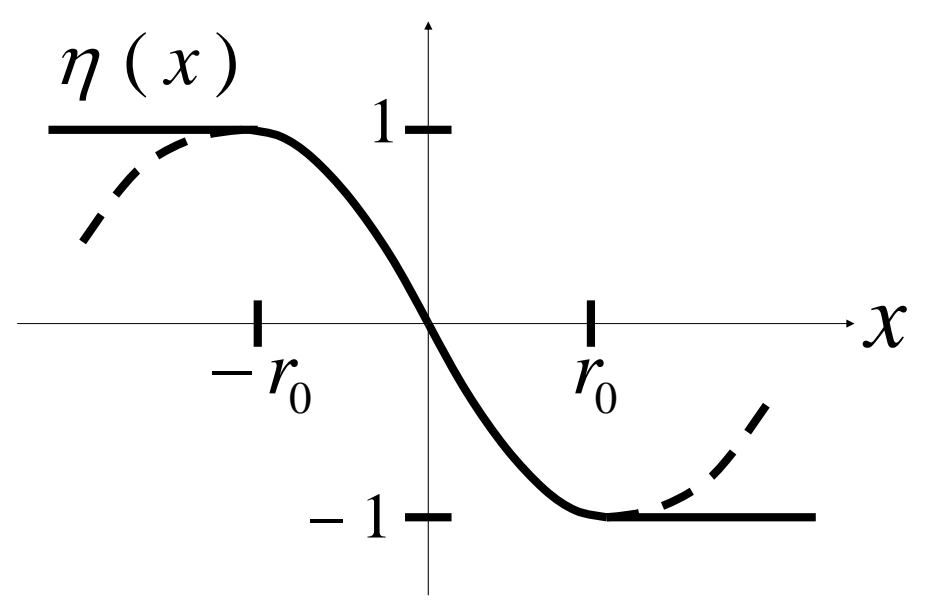

Figure 1: Profile of $\eta(x)$.

polynomial of degree 3 satisfying boundary conditions and constraint it must be the unique minimizer.

If we take

$$
\bar{\eta}(x)=a x^{3}-b x
$$

with $a=4 / L^{3}, b=3 / L$, then $\bar{\eta}$ takes its local maximum value 1 at $-r_{0}$ and its local minimum value -1 at $r_{0}$ so that $\bar{\eta}_{x}\left( \pm r_{0}\right)=0$. Of course, $|\bar{\eta}(x)| \leq 1$ is fulfilled (Figure 1). So this $\bar{\eta}$ is the minimizer of our obstacle problem. We differentiate

$$
\bar{f}= \begin{cases}-\bar{\eta}_{x}, & |x| \leq r_{0} \\ 0, & r_{0}<|x| \leq \omega / 4\end{cases}
$$

twice (in distribution sense) to get

$$
F=-\bar{f}_{x x}=\frac{24}{L^{3}} \chi_{\left(-r_{0}, r_{0}\right)}-\frac{12}{L^{2}}\left(\delta\left(x+r_{0}\right)+\delta\left(x-r_{0}\right)\right) \text { in }(-\omega / 4, \omega / 4) .
$$

The reason we have a delta function is that $\bar{f}_{x}$ has a jump discontinuity at $x= \pm r_{0}$ (Figure 2). By symmetry the proof is now complete.

We are now ready to construct an explicit form of solution starting $u_{0}$ in (3.4) (with $h_{0} \geq v_{0}\left(r_{0}\right)$ ) as an initial data. Since the strictly monotone 


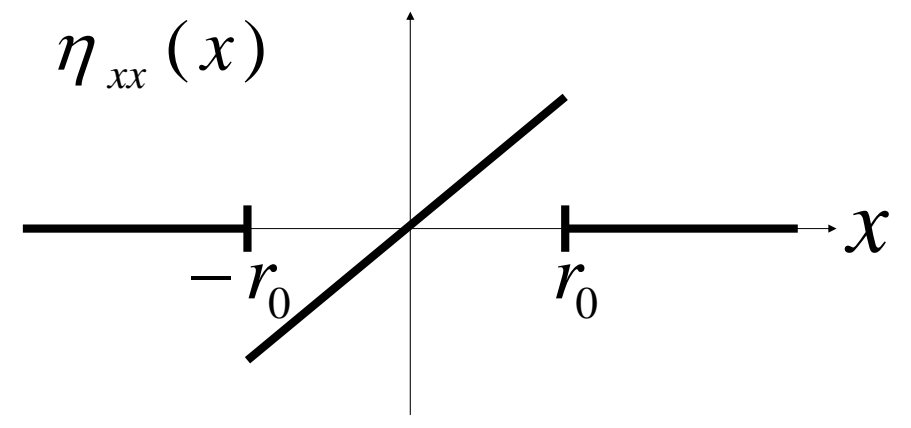

Figure 2: Profile of $\eta_{x x}(x)$.

part of $u_{0}$ does not move, it suffices to construct a solution in $(0, \omega / 4)$. We postulate a solution of the form

$$
u(x, t)=h(t) H(r(t)-x)+H(x-r(t)) v_{0}(x) \text { for } x \in(0, \omega / 4), t \geq 0,
$$

where $H$ is the Heaviside function i.e., $H(x)=1$ for $x>0$ and $H(x)=0$ for $x \leq 0$. The function $(r(t), h(t))$ satisfies the initial condition

$$
(r(0), h(0))=\left(r_{0}, h_{0}\right) .
$$

We shall derive a system of differential equations for $r(t)$ and $h(t)$. We differentiate $u$ in $t$ to get

$$
u_{t}(x, t)=\dot{h}(t) H(r(t)-x)+h(t) \delta(r(t)-x) \dot{r}(t)-v_{0}(x) \delta(r(t)-x) \dot{r}(t),
$$

where $\dot{h}$ denotes the time derivative of $h$. If $u$ solves (3.3), by Lemma 3.5 we see that $r(t)$ and $h(t)$ must satisfy

$$
\dot{h}(t)=-\frac{24}{(2 r(t))^{3}},\left(h(t)-v_{0}(r(t))\right) \dot{r}(t)=\frac{12}{(2 r(t))^{2}}
$$

or

$$
\dot{h}(t)=-3 / r(t)^{3},\left(h(t)-v_{0}(r(t))\right) \dot{r}=3 / r(t)^{2} .
$$


If $h_{0}>v_{0}\left(r_{0}\right)$, then this system of ODE has a normal form $\dot{r}=F(r, h), \dot{h}=$ $G(r, h)$ with locally Lipschitz right hand side $F, G$ near $\left(r_{0}, h_{0}\right)$, so it has a unique local-in-time solution. However, if $h_{0}=v_{0}\left(r_{0}\right)$, the quantity $h(t)-$ $v_{0}(r(t))$ is initially zero, we need more argument to construct a solution.

Proposition 3.6. Assume that $v_{0}\left(r_{0}\right)=h_{0}>0$ with $r_{0} \in(0, \omega / 4)$ and that $v_{0} \in C^{1}[0, \omega / 4]$ is strictly decreasing in the sense that $v_{0}^{\prime}<0$ in $(0, \omega / 4)$. Then there exists at least one local solution $(r(t), h(t)) \in C^{0}\left([0, \sigma), \mathbf{R}_{+}^{2}\right) \cap$ $C^{1}\left((0, \sigma), \mathbf{R}_{+}^{2}\right)$ for some $\sigma>0$ of (3.9) with initial data $\left(r_{0}, h_{0}\right)$ satisfying $h(t)-v_{0}(r(t))>0$ for $t \in(0, \sigma)$, where $\mathbf{R}_{+}=(0, \infty)$.

Proof. We transform dependent variable $r(t)$. We set $w(t)=v_{0}(r(t))$ and $k(t)=h(t)-w(t)$. Then (3.9) yields

$$
\begin{aligned}
\dot{k}(t) & =\dot{h}(t)-v_{0}^{\prime}(r(t)) \dot{r}(t) \\
& =-3 / r(t)^{3}-v_{0}^{\prime}(r(t)) 3 /\left(k(t) r(t)^{2}\right)
\end{aligned}
$$

or

$$
k \dot{k}(t)=-\frac{3 k}{r(t)^{3}}-\frac{3 v_{0}^{\prime}(r(t))}{r(t)^{2}} .
$$

Since $v_{0}^{\prime}<0$ and $k(0)=0$, the function $k^{2}$ should be increasing for small $t>0$. By definition of $w$ and $k$ we have

$$
r(t)=v_{0}^{-1}(h(t)-k(t)) .
$$

We now have a system which is equivalent to (3.9):

$$
k \dot{k}=-\frac{3 k}{\left(v_{0}^{-1}(h-k)\right)^{3}}-\frac{3 v_{0}^{\prime}\left(v_{0}^{-1}(h-k)\right)}{\left(v_{0}^{-1}(h-k)\right)^{2}}, \dot{h}=-\frac{3}{\left(v_{0}^{-1}(h-k)\right)^{3}}
$$

with initial data $k(0)=0, h(0)=h_{0}>0$. This ODE system admits a local solution by Peano's existence theorem (without uniqueness). To see this it is enough to introduce the variable $k^{2}=y$ and replace $k$ by $y_{+}^{1 / 2}$ with $y_{+}=\max (y, 0)$ to get

$$
\dot{y}=U(h, y), \dot{h}=V(h, y),
$$

where $U, V$ are continuous functions in $D=\left\{(h, y) \mid h>y_{+}^{1 / 2}, h<v_{0}(0)\right\}$ with initial data $y(0)=0, h(0)=h_{0}\left(h_{0}<v_{0}(0)\right)$. We have at least one $C^{1}$-local in time solution to (3.10) by Peano's existence theorem. Suppose 
that $y$ is a solution, then it must stay positive at least for a short time, since otherwise there is a sequence of points $\left\{t_{j}\right\}$ at which $\dot{y}\left(t_{j}\right) \leq 0$ and $y\left(t_{j}\right) \leqq 0$ with $t_{j} \downarrow 0$. However, (3.10) implies

$$
\dot{y}=-3 v_{0}^{\prime}\left(v_{0}^{-1}(h)\right) /\left(v_{0}^{-1}(h)\right)^{2}>0
$$

at such a point so we get a contradiction. So the solution we get a local $C^{1}$ solution for $(y, h)$ with the property that $y(t)>0$ for a short time $t>0$. Thus we are able to transform into the original variable to get the desired result.

Proof of Theorem 3.4. By Proposition 3.6 we have constructed a desired solution $u$ of (3.1) by giving $r(t)$ and $h(t)$ in the form of $(3.8)$ (Figures 3,4). By uniqueness of the solution of (3.1) we observe that the solution instantaneously develops jump discontinuities. It is not difficult to see that the local solution of (3.9) can be extended provided that $(r, h)$ are bounded and $k>0$. Actually it can be extended until $r$ tends to $\omega / 4$. It is not difficult to see that $r$ touches $\omega / 4$ in finite time. At that touching time the solution jumps at $\omega / 4$. From this time our obstacle problems for $\eta$ and $\zeta$ is related and there is no $\delta$ part for the minimal section (or canonical restriction). So the jump point stays at $\omega / 4$ and $h(t)$ is just decreasing with constant speed and vanishes in finite time. This is easy to observe by calculation of the minimal section. So the solution vanishes in finite time.

Remark. (i) Since the solution of (3.1) is unique, the solution of (3.9) should be also unique.

(ii) This example gives a sharp contrast between usual second order total variation flow (1.1) and $H^{-1}$ total variation flow (1.2). For (1.1) we have the maximum principle for $\left|u_{x}\right|$ so that $\left\|u_{x}\right\|_{L^{\infty}}(t) \leq\left\|u_{0 x}\right\|_{L^{\infty}}$. This indicates that Lipschitz continuity is preserved. By an extended theory of viscosity solutions [GG98Ar] we know that the continuity is also preserved. For (1.2) the solution may develop jump discontinuity instantaneously even if initial data is Lipschitz. Since Proposition 3.6 can be extended to the case that $v_{0}^{\prime}\left(r_{0}\right)=0$ and $v_{0}^{\prime}<0$ in $\left(r_{0}, \omega / 4\right)$, one is able to conclude that the solution of (1.2) may develop jump discontinuity instantaneously even if the initial data is smooth.

(iii) The development of jump discontinuity relates to the coercivity of the integrand of our energy $\Phi_{\pi}$, which is formally written as

$$
\Phi_{\pi}(u)=\int_{\mathbf{T}} e\left(u_{x}\right)
$$




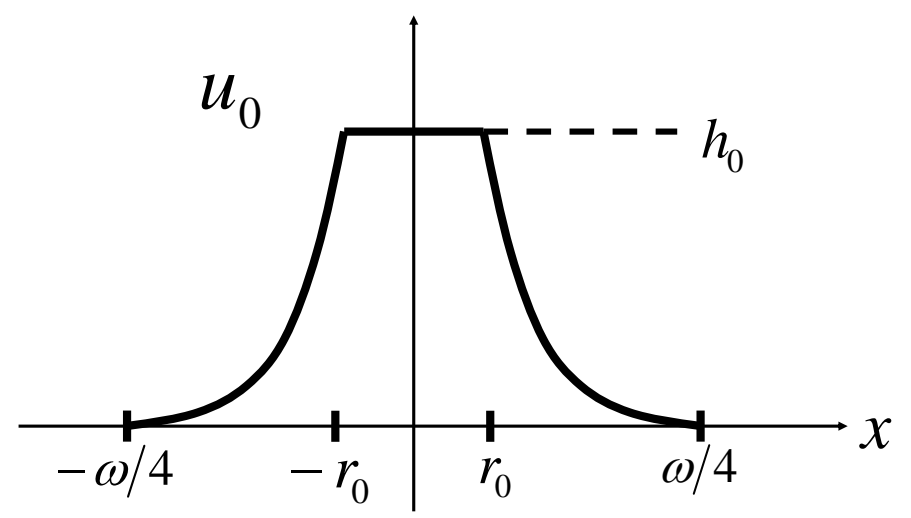

Figure 3: Profile of initial data $u_{0}$.

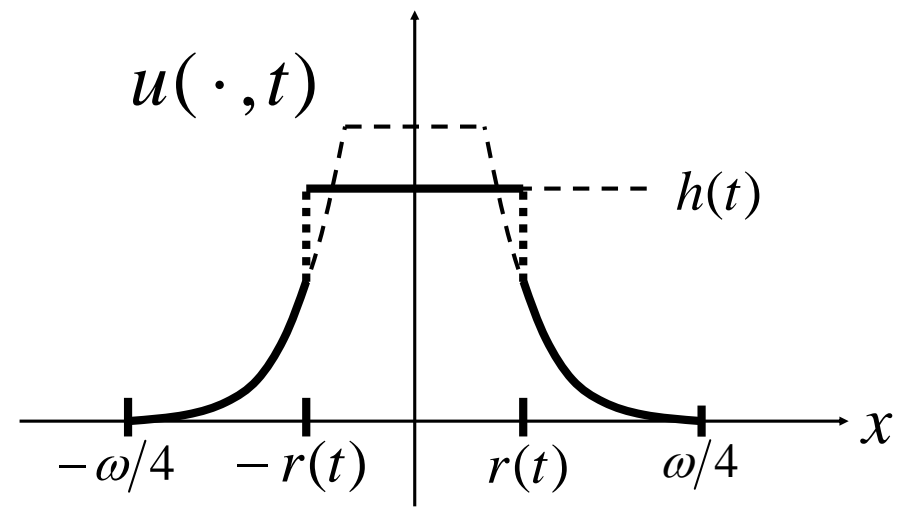

Figure 4: Profile of $u(\cdot, t)$. 
with $e(p)=|p|$. The energy density function $e(p)$ is a linear growth i.e. $\varlimsup_{p \rightarrow \infty} e(p) /|p|<\infty$. If the energy density $e(p)$ is coercive (i.e. $\lim _{p \rightarrow \infty} e(p) /|p|=$ $\infty)$ and convex, then

$$
\Phi(u)=\int e\left(u_{x}\right)<\infty
$$

implies that $u$ is (absolutely) continuous. So the solution of $u_{t} \in-\partial_{H^{-1}} \Phi(u)$ must be spatially continuous.

A typical example is the case when

$$
e_{q}(p)=|p|+\frac{\mu}{q}|p|^{q}
$$

with $q>1, \mu>0$. An $H^{-1}$ gradient flow for this energy density with $q=3$ is first introduced by $\mathrm{H}$. Spohn [Sp] to explain relaxation dynamics of a crystal surface. The subdifferential of $\Phi$ in $H^{-1}$ is calculated in [Ka]. This problem is further studied by $[\mathrm{O}]$ numerically with some analysis where minimal section is calculated. It turns out that minimal section does not include $\delta$ function for $e_{q}$.

(iv) A general upper bound for extinction time is given in [GKohn]. In fact, the extinction time $T^{*}\left(u_{0}\right)$ is estimated for example as

$$
T^{*}\left(u_{0}\right) \leq C\left\|u_{0}\right\|_{H^{-3}}^{3 / 4}\left\|u_{0}\right\|_{H^{-1}}^{1 / 4}
$$

In [GKohn] a more general estimate is given including higher dimensional problem whose dimension $n \leq 4$. It seems to be an open problem whether the solution vanishes in finite time for $n \geq 5$.

\subsection{Violation of Comparison Principle}

For such a purpose it is more convenient to consider the Dirichlet problem for (1.2) which is formally written as

$$
u_{t}=-\left(\operatorname{sgn} u_{x}\right)_{x x x} \text { in }(a, b) \times(0, \infty)
$$

with

$$
u=\left(\operatorname{sgn} u_{x}\right)_{x}=0 \quad \text { at } x=a, b .
$$

As in $[\mathrm{GGK}]$ or $[\mathrm{GKohn}]$ we consider an energy functional

$$
\Phi_{D}(u)= \begin{cases}\int_{\mathbf{R}}\left|\widetilde{u}_{x}\right|, & \tilde{u} \in B V(\mathbf{R}) \\ \infty, & \text { otherwise }\end{cases}
$$


for $u \in H^{-1}(I)$ with $I=(a, b)$. Here $\tilde{u}$ is the zero extension of $u$ outside $I$ and $H^{-1}(I)$ is the dual space of $H_{0}^{1}(I)$ which is the closure of $C_{0}^{\infty}(I)$ in $H^{1}$ space. The space $H^{-1}(I)$ is the Hilbert space equipped with an inner product

$$
((f, g))_{-1}=\left\langle\left(-\Delta_{D}\right)^{-1} f, g\right\rangle\left(=\int_{a}^{b}\left(-\Delta_{D}\right)^{-1} f \cdot g d x\right)
$$

where $-\Delta_{D}$ denotes an isometry from $H_{0}^{1}(I)$ to $H^{-1}(I)$ defined by $f \mapsto$ $((f, \cdot))_{1}$. It is regarded as the Laplacian with the Dirichlet boundary condition. Here $((f, g))_{1}=\int_{a}^{b} f_{x} g_{x} d x$ is an equivalent inner product of $H_{0}^{1}(I)$.

The problem (3.11) and (3.12) is formulated as an

$$
\begin{aligned}
& \frac{d u}{d t}(t) \in-\partial_{H^{-1}(I)} \Phi_{0}(u(t)), \text { a.e. } t>0, \\
& \left.u\right|_{t=0}=u_{0} \in H^{-1}(I),
\end{aligned}
$$

where $\partial_{H^{-1}(I)}$ denotes the subdifferential in $H^{-1}(I)$.

We now consider a special initial data $u_{0}$ defined in (3.4) with $I=$ $(-\omega / 4, \omega / 4)$. Then we observe that the solution $u$ of $(3.1)$ is also the solution of (3.13) if we restrict the domain of $u$ in $I$. For this special solution it is easy to verify that the speed belongs to $-\partial_{H^{-1}(I)} \Phi_{0}(u(t))$ at each time so that it solves (3.13). Note that the Dirichlet condition is violated from the time when $r$ hits $\omega / 4$ until the extinction time comes but this is the solution. The next theorem shows that the comparison principle is violated. We consider initial data $u_{0}$ of the form $(3.4)$ in $I=(-\omega / 4, \omega / 4)$. We fix $v_{0}$ but consider several $\left(r_{0}, h_{0}\right)$ satisfying $v_{0}\left(r_{0}\right)=h_{0}$. To clarify the dependence we shall write $u_{0}(x), u_{0}$ by $u_{0}\left[h_{0}\right](x), u_{0}\left[h_{0}\right] ; r_{0}$ is determined by $h_{0}$ by $v_{0}\left(r_{0}\right)=h_{0}$.

Theorem 3.7. Let $u\left[h_{0}\right]$ be the solution of (3.13) with initial data $u_{0}\left[h_{0}\right]$. Assume that $0<h_{1}<h_{2}<v_{0}(0)$ so that $u_{0}\left[h_{1}\right] \leq u_{0}\left[h_{2}\right]$. Then $u\left[h_{1}\right](x, t)>$ $u\left[h_{2}\right](x, t)$ for $(x, t)$ satisfying $x \in\left(v_{0}^{-1}\left(h_{1}(t)\right), r_{1}(t)\right)$ for sufficiently small $t>0$ where $\left(r_{1}(t), h_{1}(t)\right)$ is the solution of (3.9) with initial data $\left(r_{1}, h_{1}\right)$ satisfying $v_{0}\left(r_{1}\right)=h_{1}$.

Proof. By Theorem 3.4 and the form of a solution (3.8) with Proposition 3.6 we observe that $u\left[h_{1}\right](x, t)>v_{0}(x)$ for $t>0$ provided that $x \in$ $\left(v_{0}^{-1}\left(h_{1}(t)\right), r_{1}(t)\right)$. Since $u\left[h_{2}\right](x, t)=v_{0}(x)$ for sufficiently small $t>0$ near $x=r_{1}(0)$, we observe that $u\left[h_{1}\right](x, t)>u\left[h_{2}\right](x, t)$ for $x \in\left(v_{0}^{-1}\left(h_{1}(t)\right), r_{1}(t)\right)$. 
Note that by Proposition 3.6 the interval $\left(u_{0}^{-1}\left(h_{1}(t)\right), r_{1}(t)\right)$ is not empty for sufficiently small $t>0$.

\section{A Few Words about Motion by Surface Dif- fusion}

A crystalline curvature flow is considered an anisotropic version of a curve shortening equation $V=k$. The corresponding fourth order model of the mean curvature flow equation $V=H$ is the surface diffusion equation $V=$ $-\Delta_{\Gamma} H$. Compared with the mean curvature flow it is less studied since the system does not enjoy maximum principle nor comparison principle.

It is known that there is a local smooth solution for smooth initial and that an equilibrium shape-a sphere is locally stable in this flow [EMS]. However, solution may create self-intersection [GI1]. Moreover, the solution may lose convexity in finite time [GI2]. Even if initial curve is the graph of a function, it may be overturn in finite time [EM], [C]. There is an interesting work [BBW] discussing stability and self-similarity of pinch off for axisymmetric surface. This has a long list of references related to surface diffusion including $[\mathrm{CT}],[\mathrm{M}]$. Among them we just point out [CEN] where they formally derived the surface diffusion equations from a degenerate Cahn-Hilliard equations as singular limit. In the next paragraph we shall mention a classification of a surface with constant mean curvature which gives an equilibrium of surface diffusion equations. If the surface is closed (or compact), an equilibrium surface must be a surface of constant mean curvature because all harmonic function on a compact surface must be a constant by the maximum principle.

It is well-known that a constant mean curvature embedded (closed) hypersurface in $\mathbf{R}^{n}$ must be a sphere and is known as Alexandrov's theorem [A]. This result is recently extended for anisotropic case for smooth strictly convex interfacial energy by Y. He, H. Li, H. Ma, and J. Ge [HLMG] and by J. Zhai [Z]. The problem is that if the anisotropic curvature $\operatorname{div}_{\Gamma} \nabla_{p} \gamma(\vec{n})$ is constant everywhere on an embedded hypersurface, it is the boundary of a Wulff shape

$$
W_{\gamma}=\left\{x \in \mathbf{R}^{n} \mid x \cdot m \leq \gamma(m) \text { for all } m \text { such that }|m|=1\right\}
$$

up to dilation and translation. This problem (which is solved by [HLMG] for smooth strictly convex energy) was posed in the preprint version of [G2] and 
later by R. Morgan [Mo]. For singular interfacial energy it is known only for $n=2$, the case of curves by P. Mucha and P. Rybka [MR]. For $n \geq 2$ it is widely open. For development of this subject for strictly convex smooth energy the reader is referred to [HLMG], [KP1], [KP2] and references cited there.

Even if the surface in $\mathbf{R}^{3}$ is immersed, if it is defeomorphic to the sphere $S^{2}$, the constant anisotropic curvature surface is the boundary of the Wulff shape up to dilation and translation. This is first proved by Y. Giga and J. Zhai [GZ] where interfacial energy in close to isotropic. A general result is proved by [KP3] and [Z] recently for strictly convex smooth interfacial energy; see also [HL] for some attempt. In the latter article, several anisotropic constant mean curvature immersed surfaces having higher genus are constructed. However, it is an open problem for singular energy.

Acknowledgement. The authors are grateful to Professor Ken Shirakawa for informative remarks. The work of the first author was partly supported by the Grant-in-Aid for Scientific Research, No. 18540205, the Japan Society for the Promotion of Science (JSPS). The work of the second author was partly supported by the Grant-in-Aid for Scientific Research, No. 20654017, No. 21224001, JSPS.

\section{References}

[A] A. D. Alexandrov, Uniqueness theorems for surfaces in the large I, Vestnik Leningrad Univ., 11 (1956), 5-17.

[ATW] F. Almgren, J. R. Taylor and L. Wang, Curvature-driven flows: a variational approach, SIAM J. Control Optim., 31 (1993), 387-438.

[ABCM] F. Andreu-Vaillo, C. Ballester, V. Caselles and J. M. Mazón, The Dirichlet problem for the total variation flow, J. Funct. Anal., 180 (2001), 347-403.

[ACM] F. Andreu-Vaillo, V. Caselles and J. M. Mazón, Parabolic Quasilinear Equations Minimizing Linear Growth Functionals, Birkhäuser, Basel, (2004).

[AG] S.B. Angenent and M.E. Gurtin, Multiphase thermomechanics with interfacial structure 2 Evolution of an isothermal interface, Arch. Rational Mech. Anal., 108 (1989), 323-391. 
[AD] H. Attouch and A. Damlamian, Application des méthodes de convexité et monotone à l'étude de certaines équations quasi linéaires, Proc. Roy. Soc. Edinburgh Sect., A 79 (1977/78), 107-129.

[BFP] J. W. Barrett, X. Feng and A. Prohl, On $p$-harmonic map heat flows for $1 \leq p<\infty$ and their finite element approximations, SIAM J. Math. Anal., 40 (2008), 1471-1498.

[BCCN] G. Bellettini, V. Caselles, A. Chambolle and M. Novaga, Crystalline mean curvature flow of convex sets, Arch. Ration. Mech. Anal., 179 (2006), 109-152.

[BGN] G. Bellettini, R. Goglione and M. Novaga, Approximation to driven motion by crystalline curvature in two dimensions, Adv. Math. Sci. Appl., 10 (2000), 467-493.

[BM] G. Bellettini and L. Mugnai, Anisotropic geometric functional and gradient flows, Nonlocal and Abstract Parabolic Equations and Their Application, Banach Center Publications, 86, Warsaw, (2009),pp. 21-43.

[BN] G. Bellettini and M. Novaga, Approximation and comparison for nonsmooth anisotropic motion by mean curvature in $\mathbf{R}^{N}$, Math. Models Methods Appl. Sci., 10 (2000), 1-10.

[BNP] G. Bellettini, M. Novaga and M. Paolini, Facet-breaking for threedimensional crystals evolving by mean curvature, Interfaces Free Bound., 1 (1999), 39-55.

[BNP1] G. Bellettini, M. Novaga and M. Paolini, Characterization of facet breaking for nonsmooth mean curvature flow in the convex case, Interfaces and Free Boundaries, 3 (2001), 415-446.

[BNP2] G. Bellettini, M. Novaga and M. Paolini, On a crystalline variational problem, part II: $B V$ regularity and structure of minimizers on facets, Arch. Rational Mech. Anal., 157 (2001), 193-217.

[BBW] A. J. Bernoff, A. L. Bertozzi and T. P. Witelski, Axisymmetric surface diffusion: dynamics and stability of self-similar pinchoff, J. Statist. Phys., 93 (1998), 725-776. 
[B1] Y. Brenier, Une application de la symétrisation de Steiner aux équations hyperboliques : la méthode de transport et écroulement, C. R. Acad. Sci. Paris Sér I. Math., 292 (1981), 563-566.

[B2] Y. Brenier, Résolution d'équations d'évolution quasilinéaires en dimention $N$ d'espace à l'aide d'équations linéaires en dimension $N+1$. J. Differential Equations, 50 (1983), 375-390.

[B3] Y. Brenier, $L^{2}$ formulation of multidimensional scalar conservation laws, Arch. Ration. Mech. Anal., 193 (2009), 1-19.

[B] H. Brezis, Operatures Maximaux Monotone, North-Holland, Amsterdam (1973).

[CL] A. Chambolle nad P.-L. Lions, Image recovery via total variation minimization and related problems, Numer. Math., 76 (1997), 167-188.

[CEN] J. W. Cahn, C. M. Elliott and A. Novick-Cohen, The Cahn-Hilliard equation with a concentration dependent mobility: Motion by minus the Laplacian of the mean curvature, European J. Appl. Math., 7 (1996), 287-301.

[CT] J. W. Cahn and J. Taylor, Surface motion by surface diffusion, Acta metals. matel., 42 (1994), 1045-1063.

[C] J. Carrillo, Entropy solutions for nonlinear degenerate problems, Arch. Rational. Mech. Anal., 147 (1999), 269-361

[CRCT] W. C. Carter, A. R. Roosen, J. W. Cahn and J. E. Taylor, Shape evolution by surface diffusion and surface attachment limited kinetics on completely faceted surfaces, Acta. Metall. Mater., 43 (1995), 43094323 .

[CGG] Y.-G. Chen, Y. Giga and S. Goto, Uniqueness and existence of viscosity solutions of generalized mean curvature flow equations, J. Differential Geom., 33 (1991), 749-786.

[C] K.-S. Chou, A blow-up criterion for the curve shortening flow by surface diffusion, Hokkaido Math. J., 32 (2003), 1-19.

[Chu] K.-Y. Chung, On variational schemes modeling surface diffusion, $\mathrm{PhD}$ Dissertation, Princeton University (1997). 
[CIL] M. Crandall, H. Ishii and P.-L. Lions, User's guide to viscosity solutions of second order partial differential equations, Bull. Amer. Math. Soc., 27 (1992), 1-67.

[EM] C. M. Elliott and S. Maier-Paape, Losing a graph with surface diffusion, Hokkaido Math. J., 30 (2001), 297-305.

[EIS] C. M. Elliott and S. A. Smitheman, Analysis of the TV regularization and $H^{-1}$ fidelity model for decomposing an image into cartoon plus texture, Commun. Pure Appl. Anal., 6 (2007), 917-936.

[ElS2] C. M. Elliott and S. A. Smitheman, Numerical analysis of the TV regularization and $H^{-1}$ fidelity model for decomposing an image into cartoon plus texture, IMA J. Numer. Anal., 29 (2009), 651-689.

[EMS] J. Escher, U. F. Mayer and G. Simonett, The surface diffusion flow for immersed hypersurfaces, SIAM J. Math. Anal., 29 (1998), 1419-1433.

[EFG] L.C. Evans, M. Feldman and R. F. Gariepy, Fast/slow diffusion and collapsing sandpiles, J. Differential Equations, 137 (1997), 166-209.

[ES] L.C. Evans and J. Spruck, Motion of level sets by mean curvature, I, J. Differential Geom., 33 (1991), 635-681.

[FG] T. Fukui and Y. Giga, Motion of a graph by nonsmooth weighted curvature, In: World Congress of Nonlinear Analysts '92 (ed. V. Lakshmikantham), Walter de Gruyter, Berlin I (1996), 47-56.

[Gia M] L. Giacomelli and S. Moll, Rotationally symmetric 1-harmonic flows $D^{2}$ to $S^{2}$ local well-posedness and finite time blow up, preprint.

[GG98Ar] M.-H. Giga and Y. Giga, Evolving graphs by singular weighted curvature, Arch. Rational Mech. Anal., 141 (1998), 117-198.

[GG98DS] M.-H. Giga and Y. Giga, A subdifferential interpretation of crystalline motion under nonuniform driving force, In: Proc. of the International Conference in Dynamical Systems and Differential Equations, Springfield Missouri, (1996), Dynamical Systems and Differential Equations (eds. W.-X. Chen and S.-C. Hu), Southwest Missouri Univ. 1 (1998), pp.276-287. 
[GG99] M.-H. Giga and Y. Giga, Stability for evolving graphs by nonlocal weighted curvature, Commun. in Partial Differential Equations, 24 (1999), 109-184.

[GG01Ar] M.-H. Giga and Y. Giga, Generalized motion by nonlocal curvature in the plane, Arch. Rational Mech. Anal., 159 (2001), 295-333.

[GG] M.-H. Giga and Y. Giga, A PDE approach for motion of phaseboundaries by a singular interfacial energy, Tochastic Analysis on Large Scale Interacting System, Adcanced Studies in Pure Math., 39 (eds. T. Funaki and H. Osada), (2004), Math Soc. Japan, pp. 213-232.

[GG03] M.-H. Giga and Y. Giga, Minimal vertical singular diffusion preventing overturning for the Burgers equation, Recent Advances in Scientific Computing and Partial Differential Equations, Contemp. Math. 330 (2003), Amer. Math. Soc. Providence, pp.73-88.

[GGH] M.-H. Giga, Y. Giga and H. Hontani, Self-similar expanding solutions in a sector for a crystalline flow, SIAM J. Math. Anal. 37 (2005), 12071226.

[GGO] M.-H. Giga, Y. Giga and Y. Ochiai, A crystalline flow starting from a general polygon, in preparation.

[GGK] M.-H. Giga, Y. Giga and R. Kobayashi, Very singular diffusion equations, Adv. Studies in Pure Math 31, Taniguchi Conference on Mathematics Nara 98, (2001), (eds. M. Maruyama and T. Sunada), pp.93-125.

[GGiR] M.-H. Giga, Y. Giga and P. Rybka, A comparison principle for singular diffusion equations with spatially inhomonegeous driving force, in preparation.

[G0] Y. Giga, Anisotropic curvature effects in interface dynamics, Sugaku Expositions, 16, (2003), 135-152. (translation from Sūgaku 52 (2000), 113-127.)

[G1] Y. Giga, Singular diffusivity - Facets, Shocks anda more, Applied Math Entering the 21th Century, eds J. M. Hill and R. Moore, ICIAM 2003 Sydney (2004), pp.121-138, SIAM, Philadelphia. 
[G2] Y. Giga, Viscosity solutions with shocks, Comm. Pure Appl. Math., 55 (2002), 431-480.

[G3] Y. Giga, Surface Evolution Equations - a level set approach, Birkhäuser (2006).

[GGM] Y. Giga, M. E. Gurtin and J. Matias, On the dynamics of crystalline motions, Japan J. Indust, Appl. Math., 151 (1998), 7-50.

[GGR] Y. Giga, P. Górka and P. Rybka, Nonlocal spatially inhomogeneous Hamilton-Jacobi equation with unusual free boundary, Discrete Contin. Dyn. Syst., 26 (2010), 493-519.

[GI1] Y. Giga and K. Ito, On pinching of curves moved by surface diffusion, Comm. Appl. Anal. 2 (1998), 393-405.

[GI2] Y Giga and K. Ito, Loss of convexity of simple closed curves moved by surface diffusion, Topics in Nonlinear Analysis, The Herbert Amann Aniversary volume, (eds. J. Escher and G. Simonett), Progress in Nonlinear Differential Equations, 35, Birkhäuser, (1999), 305-320.

[GKY] Y. Giga, Y. Kashima and N. Yamazaki, Local solvability of a constrained gradient system of total variation, Abstr. Appl. Anal., (2004), 651-682.

[GK] Y. Giga and R. Kobayashi, On constrained equations with singular diffusivity, Methods Appl. Anal., 10 (2003), 253-277.

[GKohn] Y. Giga and R. V. Kohn, Scale-invariant extinction time estimates for some singular diffusion equations, in preparation.

[GKu] Y. Giga and H. Kuroda, On breakdown of solutions of a constrained gradient system of total variation, Bol. Soc. Parana. Mat., (3) 22 (2004), 9-20.

[GM] Y. Giga and T. Miyakawa, A kinetic construction of slobal solutions of first order quasilinear equations, Duke Math. J., 50 (1983), 505-515.

[GMO] Y. Giga, T. Miyakawa and S. Oharu, A kinetic approach to general first order quasilinear equations, Trans. Amer. Math. Soc., 287 (1987), 723-743. 
[GOS] Y. Giga, T. Ohtsuka and R. Schätzle, On a uniform approximation of motion by anisotropic curvature by the Allen-Cahn equations, Interfaces Free Bound., 8 (2006), 317-348.

[GR4] Y. Giga and P. Rybka, Facet bending in the driven crystalline curvature flow in the plane, J. Geom. Anal., 18 (2008), 109-147.

[GR5] Y. Giga and . Rybka, Facet bending driven by the planar crystalline curvature with a generic nonuniform forcing term, J. Differential Equations, 246 (2009), 2264-2303.

[GPR] Y. Giga, M. Paolini and P. Rybka, On the motion by singular interfacial energy, Japan J. Indust. Appl. Math., 18 (2001), 231-248.

[GZ] Y. Giga and J. Zhai, Uniqueness of constant weakly anisotropic mean curvature immersion of sphere $S^{2}$ in $\mathbf{R}^{3}$, Adv. Diff, Eq., 14 (2009), 601-619.

[HZ] R. Hardt and X. Zhou, An evolution problem for linear growth functionals, Comm. Partial Differential Equations, 19 (1994), 1879-1907.

[HLMG] Y. He, H. Li, H. Ma and J. Ge, Compact embedded hypersurfaces with constant higher order anisotropic mean curvatures, Indiana Univ. Math. J., 58 (2009), 853-868.

[HL] Y. He and H. Li, Anisotropic version of a theorem of H. Hopf, Ann. Global Anal. Geom., 35 (2009), 243-247.

[I] T. Ishiwata, Motion of non-convex polygons by crystalline curvature and almost convexity phenomena, Japan J. Indust. Appl. Math., 25 (2008), 233-253.

[IGNS] A. Ito, M. Gokieli, M. Niezgódka and M. Szpindler, Mathematical analysis of approximate system for one-dimensional grain boundary motion of Kobayashi-Warren-Carter type, preprint.

[IKY1] A. Ito, N. Kenmochi and N. Yamazaki, A phase-field model of grain boundary motion, Appl. Math., 53 (2008), 433-454.

[IKY2] A. Ito, N. Kenmochi and N. Yamazaki, Weak solutions of grain boundary motion model with singularity, Rend. Mat. Appl., (7), 29 (2009), 51-63. 
[KG] R. Kobayashi and Y. Giga, Equations with singular diffusivity, J. Stat. Phys. 95 (1999), 1187-1220.

[KWC] R. Kobayashi, J. A. Warren and W. C. Carter, A continuum model of grain boundaries, Phys. D., 140 (2000), 141-150.

[Ka] Y. Kashima, A subdifferential formulation of fourth order singular diffusion equations, Adv. Math. Sci. Appl., 14 (2004), 49-74.

[KL] B. Kawohl and Th. Lachand-Robert, Characterization of Cheeger sets for convex subsets of the plane, Pacific J. Math., 225 (2006), 103-118.

[KSch] B. Kawohl and F. Schuricht, Dirichlet problems for the 1-Laplace operator, including the eigenvalue problem, Commun. Contemp. Math., 9 (2007), 515-543.

[KS] D. Kinderlehrer and G. Stampacchia, An Introduction to Variational Inequalities and their Applications, Academic Press, New York (1980)

[KS1] R. V. Kohn and S. Serfaty, A deterministic-control-based approach to motion by curvature, Comm. Pure Appl. Math., 59 (2006), 344-407.

[KS2] R. V. Kohn and S. Serfaty, A deterministic-control-based approach to fully nonlinear parabolic and elliptic equations, preprint.

[KP1] M. Koiso and B. Palmer, Geometry and stability of surfaces with constant anisotropic mean curvature, Indiana Univ. Math. J., 54 (2005), $1817-1852$.

[KP2] M. Koiso and B. Palmer, Stability of anisotropic capillary surfaces between two parallel planes, Calc. Var. Partial Differential Equations, 25 (2006), 275-298.

[KP3] M. Koiso and B. Palmer, Anisotropic umblic points and Hopf's Theorem for surface with constant anisotropic mean curvature, preprint.

[Ko] Y. Kōmura, Nonlinear semi-groups in Hilbert space, J. Math. Soc. Japan, 19 (1967) 493-507.

[Kr] S. N. Kruzhkov, First order quasilinear equations with several independent variables, Mat. 56. (N.S.) 81. (123), (1970), 228-255 
[Mi] T. Miyakawa, Construction of solutions of a semilinear paraboric equation with the aid of the linear Boltzmann equation, Hiroshima Math. J. 14 (1984), 299-310.

[Mol] J. S. Moll, The anisotropic total variation flow, Math. Ann., 332 (2005), 177-218.

[Mo] F. Morgan, Planar Wulff shape is unique equilibrium, Proc. Amer. Math. Soc., 133 (2005), 809-813.

[MR] P. B. Mucha and P. Rybka, A new look at equilibria in Stefan-type problems in the plane, SIAM J. Math. Anal., 39 (2007), 1120-1134.

[M] W. W. Mullins, Theory of thermal groovbing, J. Appl. Phy., 28 (1957), 333-339.

[O] I. V. Odisharia, Simulation and analysis of the relaxation of a crystalline surface, PhD Thesis, New York University (2006).

[OSV] S. Osher, A. Solé and L. Vese, Image decomposition and restoration using total variation minimization and the $H^{-1}$ norm, Multiscale Model. Simul., 1 (2003), 349-370.

[ROF] L. Rudin, S. Osher and E. Fatemi, Nonlinear total variation based noise removal algorithm, Physica D., 60 (1992), 259-268.

[R] A. R. Roosen, Crystalline curvature and flat flow in a diffusion field, unpublished note (1994).

[S] K. Shirakawa, Stability for steady-state solutions of a nonisothermal Allen-Cahn equation generated by a total variation energy, Nonlinear partial differential equations and their applications, GAKUTO Internet. Ser. Math. Sci. Appl., 20, Gakkotosho, Tokyo(2004), 289-304.

[SK] K. Shirakawa and M. Kimura, Stability analysis for Allen-Cahn type equation associated with the total variation energy, Nonlinear Analysis, 60 (2005), 257-282.

[Sp] H. Spohn, Surface dynamics below the roughening temperature, J. Phys. I. France, 3 (1993), 69-81. 
[TSC] B. Tang, G. Sapiro and V. Caselles, Diffusion of general data on nonflat manifold via harmonic map theory; the direction diffusion case, Int. J. Comp. Vision 36 (2000), 149-161.

[T1] J. Taylor, Constructions and conjectures in crystalline nondifferential geometry, In: Differential geometry, (eds. B. Lawson and K. Tanenblat), Proceedings of the Conference on Differential Geometry, Rio de Janeiro, Pitman Monographs in Pure and Applied Math., 52 (1991), pp. 321-336, Pitman, London.

[TGO] Y.-H. R. Tsai, Y. Giga and S. Osher, A level set approach for computing discontinuous solutions of Hamilton-Jacobi equations, Math. Comp., 72 (2003), 159-181.

[VO] L. Vese and S. Osher, Numerical methods for $p$-harmonic flows and applications to image processing, SIAM J. Numer. Anal., 40 (2002), 2085-2104.

[WO] H. Watanabe and S. Oharu, BV-entropy solutions to nonlinear strongly degenerate parabolic equations, Advances in Differential Equations, 15 (2010), 757-800.

[Z] J. Zhai, On the uniqueness conjecture of Wulff shape, preprint. 